\title{
Coexistence of vitreomacular traction and exudative age-related macular degeneration. A case report
}

\author{
Katarzyna Michalska-Małecka', ${ }^{1,}$, Michał Chrząszcz², Katarzyna Witek ${ }^{2}$ \\ 'Department of Ophthalmology, School of Medicine in Katowice, Medical University of Silesia, Katowice, Poland \\ ${ }^{2}$ University Clinical Centre, University Hospital Medical University of Silesia, Katowice, Poland
}

\begin{abstract}
Vitreomacular traction is a common phenomenon causing a decrease in visual acuity, metamorphopsia, and disorders of central vision. The introduction of intravitreal ocriplasmin injection provided a new possibility of noninvasive treatment of this syndrome. The novelty of this case is that the ocriplasmin is a new treatment possibility and its combination with ranibizumab treatment has rarely been reported. The aim was to report a case of an 83-year-old man treated with intravitreal ocriplasmin and ranibizumab injections because of coexisting vitreomacular traction and exudative age-related macular degeneration.

A patient was referred to our clinic due to complaints about progressive central visual acuity decrease and metamorphopsia in the right eye observed within the last year. Before intravitreal $125-\mu \mathrm{g}$ ocriplasmin injection the patient underwent ophthalmic examinations including best corrected visual acuity, slit-lamp, and fundoscopy and optical coherence tomography. After diagnosing with exudative age-related macular degeneration the patient was referred to three loading doses of anti-VEGF therapy. Three weeks after ocriplasmin injection the patient's best corrected visual acuity improved. Ophthalmic examinations revealed the occurrence of exudative age-related macular degeneration. After three loading doses of anti-VEGF therapy ophthalmic examinations disclosed vitreomacular traction release and retinal fluid absorption.

Coexistence of vitreomacular diseases may be the reason for poor response to anti-VEGF treatment in patients with exudative age-related macular degeneration. Simultaneously, pharmacological vitreolysis using ocriplasmin injection may be an effective and safe method of vitreomacular traction treatment.
\end{abstract}

KEY WORDS: vitreomacular traction, ocriplasmin, metamorphopsia, age-related macular degeneration, ranibizumab

\section{INTRODUCTION}

Ageing changes occur in all structures of the eye. Common phenomenon frequently related to aging of ocular structures is posterior vitreous detachment (PVD). As we age, the vitreous gel liquefies and contracts away from its posterior adhesion at the retina, optic disk, and retinal vessels. Studies have showed that the posterior vitreous detachment is detected in half of 50-year-old subjects and almost in all subjects aged 80 years or older [1].
Incomplete posterior vitreous detachment may cause vitreomacular traction (VMT). This frequent macular condition causes anatomical changes in the contour of the foveal surface, intraretinal pseudocyst formation, and disappearance of foveal depression, which results in reduced or distorted vision [2-3].

The prevalence of vitreomacular traction has been estimated as approximately 22.5 cases per 100,000 of the general population, with an incidence of 0.6/100,000 persons-year [4]. 

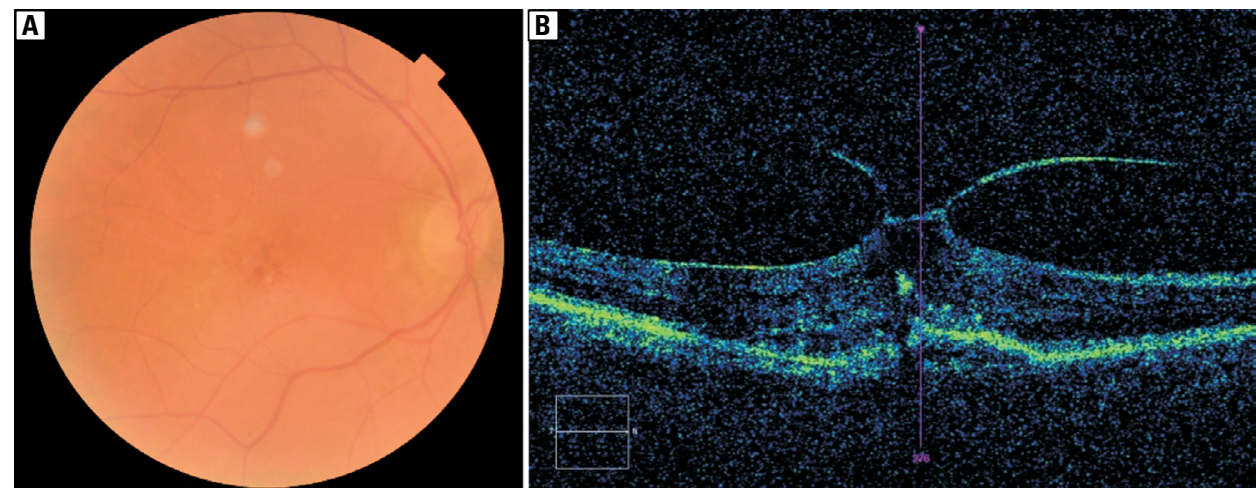

FIGURE 1. Fundoscopy of right eye. Vitreous condensation in macula region and drusen (A); Right eye. OCT scan before ocriplasmin injection. Focal vitreomacular traction with intraretinal cysts. Retinal distortion caused by abnormal traction forces on macula by the posterior vitreous face. Hyporeflective space under the neurosensitive retina - eAMD suspicion (B)

Development of non-invasive high-resolution optical coherence tomography (OCT) imaging has provided new insight into the understanding of vitreomacular traction syndrome by providing better evaluation of the tractional forces at the vitreoretinal interface, as well as recognising its relationship with particular macular conditions.

Nowadays, VMT is believed to be associated with a broad spectrum of maculopathies, including cystoid macular oedema (CMO), epiretinal membrane (ERM), and macular hole (MH) formation, all attributed to a common aetiology [5-6].

Krebs and Schulze suggested that vitreoretinal interface abnormalities, specifically vitreomacular traction, may be a potential risk factor in the development of exudative age-related macular degeneration (eAMD) [7-9]. The current standard care for neovascular age-related macular degeneration is anti-VEGF therapy through anti-VEGF injections.

We would like to present a case of VMT accompanied by eAMD and a novel approach to the treatment of coexistence of these diseases. Ocriplasmin is not the only innovative therapeutic agent. Also, very rarely clinicians decide to use both agents simultaneously. The presented case confirmed the effectivity of combining two therapies.

\section{MATERIALS, METHODS, AND RESULTS}

An 83-year-old man was referred to our clinic due to complaints about progressive central visual acuity decrease and metamorphopsia in the right eye observed within the last year. The previous medical history was unremarkable except for hypertension and cataract extraction using phacoemulsification with intraocular lens implantation in the left eye. After the treatment the patient's best cor- rected visual acuity (BCVA) increased from 0.3 to 0.7 . The right eye had not been subjected to any treatment previously.

At the initial visit, the BCVA was 0.2 in the right eye and 0.7 in the left eye. Ophthalmological examination using slit-lamp and fundoscopy revealed cortico-nuclear cataract, vitreous condensation in the macula region and multiple drusen in the right eye (Fig. 1A). The left eye was pseudophakic, with posterior capsule opacity. The fundoscopy view suggested macular hole.

Spectral-Domain OCT demonstrated foveal vitreomacular traction with marked cystic distortion of the central retina in the right eye and hyporeflective space under neurosensory retina - eAMD suspicion (Fig. 1B). Lamellar macular hole with total posterior vitreous detachment and confluent hard drusen were detected in the left eye.

B-scan ultrasound of right eye revealed partial PVD with focal VMT attached to the region of the macula.

The patient was qualified for intravitreal injection of $125 \mu \mathrm{g}$ ocriplasmin (Jetrea ${ }^{\circledR}$; ThromboGenics, Iselin, N.J., USA) to the right eye. Before ocriplasmin treatment the patient gave formal written consent. The patient's treatment was part of a study approved by the Bioethical Committee of the Medical University of Silesia (approval number $\mathrm{KNW} / 0022 / \mathrm{KB} 1 / 13 / \mathrm{I} / 15)$ and was conducted in accordance with the Declaration of Helsinki. It was assumed that the treatment would allow to stabilisation or improvement of patient's vision. Moreover, it was predicted that the applied treatment would prevent further destruction of the retina resulting from VMT mechanical pull on the retina as well as the unfavourable impact of neovascular age-related macular degeneration. 
Three weeks after this injection the BCVA in his right eye improved to 0.4 . Follow-up OCT still showed VMT with less intensity of the traction in the fovea region with smaller cystic spaces. Additionally, subretinal fluid and focal hyperreflective space in the retina was shown. The scans suggested intercurrent active choroidal neovascularisation (CNV) - exudative age-related macular degeneration (eAMD) (Fig. 2A). Fluorescein angiography confirmed CNV - exudative age-related macular degeneration (Fig. 2B).

Due to diagnosis of eAMD the patient was referred for three loading doses of anti-VEGF therapy. One week later the patient underwent a first intravitreal injection of $0.5 \mathrm{mg}$ ranibizumab (Lucentis; Novartis, Basel, Switzerland, and Genentech, Inc., South San Francisco, Calif., USA). At a follow-up visit four weeks later the BCVA was 0.4. OCT revealed a decrease in subretinal fluid. Residual VMT was still observed. Three days later a second dose of ranibizumab was injected into the right eye.

One week after the second injection of ranibizumab, the best corrected visual acuity of the right eye improved to 0.5 . OCT showed complete VMT release. Subretinal fluid was not detected in the treated eye (Fig. 2C). Three weeks later the patient underwent a third loading ranibizumab injection. In the control examination OCT scans were stable - there was no fluid under neurosensory retina. BCVA improved to 0.7 .

During the treatment no adverse events were observed. The treatment was well tolerated by the patient.

It should be emphasised that the patient expressed high satisfaction with the therapy results, connected with visual acuity improvement and overcoming troubling symptoms in the form of metamorphopsia and micro- and macropsia.

It is believed that the novelty and effectiveness of the applied treatment method are undoubted strengths of the approach to this case. Moreover, an advantage is the individualisation of the approach.

\section{DISCUSSION}

VMT was first described and confirmed by histological studies in 1970 by Reese et al. [10].

Development of noninvasive OCT imaging in the last two decades has provided new insight into VMT - our understanding of the pathophysiology of this condition and its role in the pathogenesis of other symptoms of the retina.
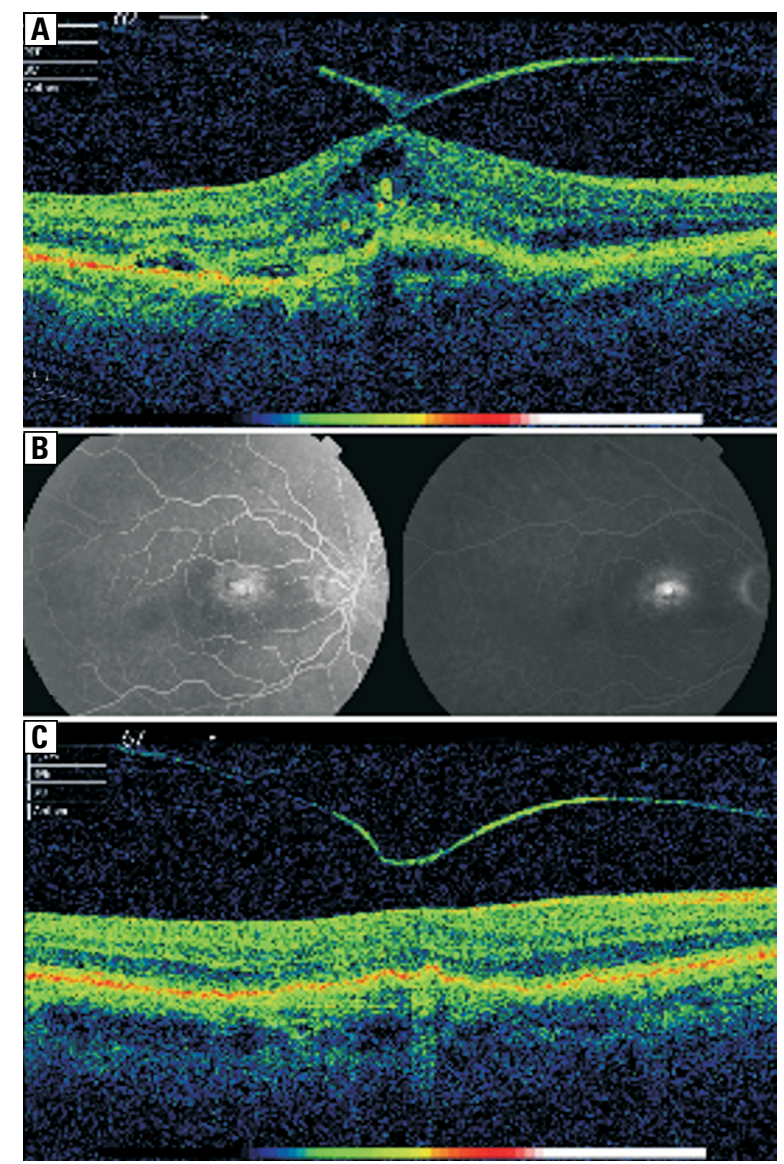

FIGURE 2. RE OCT scan 3 weeks after ocriplasmin injection. Dissolving vitreomacular traction with foveal cystoids changes involving the inner retina. Subretinal fluid (A); RE FA. An area of hyperfluorescence identified in the early phase of angiogram, which progressively intensifies throughout the transient phase, with leakage of dye (B); RE OCT scan. One week after second ranibizumab injection. Vitreomacular traction release. Absence of subretinal fluid (C)

Typical symptoms reported by patients with vitreomacular traction are: decrease of visual acuity, metamorphopsia, and disorders of central vision [5].

Until now, treatment of symptomatic vitreomacular traction causing the decrease of visual acuity and the disrupting layer structure of the retina was the pars plana vitrectomy procedure, which is burdened with the risk of severe intraoperative complications. Clinicians made the decision to carry our pars plana vitrectomy when visual acuity was below 5/16.

The introduction of intravitreal ocriplasmin injection provided a new possibility of noninvasive treatment [11].

Ocriplasmin is a truncated form of human plasmin produced by DNA recombination methods. It is characterised by strong proteolytic activity to- 
wards vitreous body protein and the connection between the vitreous body and the retina.

Through lamin and fibronectin digestion, collagen leads to enzymatic vitreolysis - dissolution of protein matrix, which is the cause of the abnormal adhesion of the vitreous body to the retina [12-15].

In phase III of the MIVI-TRUST trials Stelsmans et al. proved that in comparison to placebo, ocriplasmin application (in a dose of $125 \mu \mathrm{g}$, in a volume of $0.1 \mathrm{~mL}$ ) in adults with symptomatic vitreomacular traction, after 28 days observation period, increased the probability of vitreomacular adhesion (VMA) detachment and macular hole closure without the need of surgical intervention. Pharmacological VMT detachment was observed in $26.5 \%$ of eyes after ocriplasmin injection in comparison to $10.1 \%$ of eyes after placebo $(\mathrm{p}<0.001)$. Full posterior vitreous detachment was more frequent in eyes treated with ocriplasmin (13.4\%) than in eyes treated with placebo (3.7\%). Nonsurgical closure of macular hole was observed in $40.6 \%$ of eyes after ocriplasmin injection as compared to $10.6 \%$ of eyes after placebo injection [16].

Simultaneously, after ocriplasmin injection floaters may occur in the vitreous body. Moreover, the injection increased the probability of complications in the form of photopsia, conjunctival bleeding, eye pain caused by injection, blurred vision and vision disturbances without increasing the risk of severe complications including macular hole formation, retinal detachment, and visual acuity decrease [16].

At the Cole Clinic 33\% (8/17) of patients achieved VMA resolution, and 80\% (4/5) had closure of their full thickness macular hole (FTMH) after ocriplasmin injection [17].

Krebs and Schulze suggested that vitreoretinal interface abnormalities, specifically vitreomacular traction, may be a potential risk factor in the development of exudative age-related macular degeneration [7-9]. In the literature we can find numerous hypotheses trying to explain how the diseases of vitreoretinal junction influence neovascularisation development. The most common cause mentioned in the literature is induced secretion of proangiogenic factors (VEGF) in the course of these diseases [18-19]. The mechanical distortion of RPE caused by forces affecting the retina in the course of VMT may stimulate the retinal pigment epithelium to secrete VEGFs as a response to mechanical stress [20-21].

Additionally, it is believed that vitreous body detachment is associated with better retinal oxidation, and that adhered vitreous body decreases it. Retinal hypoxia is a well-known potential factor stimulating the secretion of vascular growth factors [22].

This may explain worse response to anti-VEGF treatment in patients with neovascular AMD and comorbid diseases of vitreoretinal space.

\section{CONCLUSIONS}

The reason for poor response to anti-VEGF treatment in patients with neovascular AMD might be coexisting pathologies of vitreoretinal junctions. An effective and safe method of symptomatic vitreomacular traction treatment is pharmacological vitreolysis with the use of intravitreal ocriplasmin injection. It is also an excellent example of the prevailing trend in modern ophthalmology - minimising the invasiveness of medical procedures.

Favourable examination outcomes after the treatment confirmed the legitimacy of ocriplasmin and ranibizumab application in patients with coexisting vitreomacular traction and neovascular age-related macular degeneration.

\section{Conflict of interest}

The authors declare that they have no competing interests. This research did not receive any specific grant from funding agencies in the public, commercial, or not-for-profit sectors.

\section{REFERENCES}

1. Sebag J. Age-related changes in human vitreous structure. Graefes Arch Clin Exp Ophthalmol 1987; 225: 89-93.

2. Carrero JL. Incomplete posterior vitreous detachment: prevalence and clinical relevance. Am J Ophthalmol 2012; 153: 497-503. doi: 10.1016/j.ajo.

3. García-Layana A, García-Arumí J, Ruiz-Moreno JM, Arias-Barquet L, Cabrera-Lopez F, Figueroa MS. A review of current management of vitreomacular traction and macular hole. J Ophthalmol 2015; 2015: 809640. doi: $10.1155 / 2015 / 809640$.

4. Jackson TL, Nicod E, Simpson A, Angelis A, Grimaccia F, Kanavos P. Symptomatic vitreomacular adhesion. Retina 2013; 33: 1503-1511. doi: 10.1097/IAE.0b013e31829232fd.

5. Bottós J, Elizalde J, Arevalo JF, Rodrigues EB, Maia M. Vitreomacular traction syndrome. J Ophthalmic Vis Res 2012; 7: 148-161.

6. Johnson MW. Posterior vitreous detachment: evolution and complications of its early stages. Am J Ophthalmol 2010; 149: 371-382. doi: 10.1016/j.ajo.2009.11.022.

7. Shulze S, Neugebauer A, Kroll P. Appearance of age-related macular degeneration in vitrectomized and nonvitrectomized eyes: an intraindividual case study. Acta Ophthalmol 2012; 90: 244-247. doi: 10.1111/j.1755-3768.2010.01929.x.

8. Krebs I, Brannath W, Glittenberg C, Zeiler F, Sebag J, Binder S. Posterior vitreomacular adhesion: a potential risk factor for exudative age-related macular degeneration? Am J Ophthalmol 2007; 144: 741-746. doi: 10.1016/j.ajo.2007.07.024.

9. Krebs I, Glittenberg C, Zeiler F, Binder S. Spectral domain optical coherence tomography for higher precision in the evaluation of vitreoretinal adhesions in exudative age-related macular degeneration. $\mathrm{Br}$ J Ophthalmol 2011; 95: 1415-1418. doi: 10.1136/bjo.2010.192385. 
10. Reese $A B$, Jones IS, Cooper WC. Vitreomacular traction syndrome confirmed histologically. Am J Ophthalmol 1970; 69: 975-977.

11. Kuppermann $\mathrm{BD}$. Ocriplasmin for pharmacologic vitreolysis. Retina 2012; 32: 228-231. doi: 10.1097/IAE.0b013e31825bc593.

12. De Smet MD, Valmaggia C, Zarranz-Ventura J, Willekens B. Microplasmin: ex vivo characterization of its activity in porcine vitreous. Invest Ophthalmol Vis Sci 2009; 50: 814-819. doi: 10.1167/iovs.08-2185.

13. Gandorfer A. Microplasmin-assisted vitrectomy. Dev Ophthalmol 2009; 44: 26-30. doi: 10.1159/000223942.

14. Gandorfer A, Rohleder M, Sethi $C$ et al. Posterior vitreous detachment induced by microplasmin. Invest Ophthalmol Vis Sci 2004; 45: 641-647.

15. Chin EK, Almeida DR, Sohn EH et al. Incomplete vitreomacular traction release using intravitreal ocriplasmin. Case Rep Ophthalmol 2014; 12; 5: 455-462. doi: 10.1159/000370024.

16. Stalmans $P$, Benz MS, Gandorfer A et al. Enzymatic vitreolysis with ocriplasmin for vitreomacular traction and macular holes. N Engl J Med 2012; 367: 606-615. doi: 10.1056/NEJMoa1110823.

17. Singh RP, Li A, Bedi R et al. Anatomical and visual outcomes following ocriplasmin treatment for symptomatic vitreomacular traction syndrome. Br J Ophthalmol 2014; 98: 356-360. doi: 10.1136/bjophthalmol-2013-304219.

18. Krishnan R, Arora R, De Salvo $G$ et al. Vitreomacular traction affects anti-vascular endothelial growth factor treatment outcomes for exudative age-related macular degeneration. Retina 2015; 35: 1750-1756. doi: 10.1097//AE.0000000000000714.

19. Mojana F, Cheng L, Bartsch DU et al. The role of abnormal vitreomacular adhesion in age-related macular degeneration: spectral optical coherence tomography and surgical results. Am J Ophthalmol 2008; 146: 218-227. doi: 10.1016/j.ajo.2008.04.027.

20. Seko Y, Seko Y, Fujikura H, Pang J, Tokoro T, Shimokawa H. Induction of vascular endothelial growth factor after application of mechanical stress to retinal pigment epithelium of the rat in vitro. Invest Ophthalmol Vis Sci 1999; 40: 3287-3291.

21. Simpson AR, Petrarca R, Jackson TL. Vitreomacular adhesion and neovascular age-related macular degeneration. Surv Ophthalmol 2012; 57: 498-509. doi: 10.1016/j.survophthal.2012.01.011.

22. Minchenko A, Bauer T, Salceda S. Hypoxic stimulation of vascular endothelial growth factor expression in vitro and in vivo. Lab Invest 1994; 71: 374-379. 
